\title{
Agrobiodiversidade nos quintais e roçados ribeirinhos na comunidade Boca do Môa - Acre
}

\author{
Williane Maria de Oliveira Martins ${ }^{1 *}$ \\ Lilliane Maria de Oliveira Martins ${ }^{1}$ \\ Fabiano Silveira Paiva ${ }^{1}$ \\ Wilton José de Oliveira Martins ${ }^{2}$ \\ Sebastião Ferreira Lima Júnior ${ }^{1}$ \\ ${ }^{1}$ Instituto Federal do Acre \\ Avenida 25 de Agosto, 2231, CEP 69980-000, Cruzeiro do Sul - AC, Brasil \\ ${ }^{2}$ Universidade Federal do Acre \\ * Autor para correspondência \\ williane.martins@ifac.edu.br
}

Submetido em 04/11/2011

Aceito para publicação em 08/06/2012

\section{Resumo}

Os roçados e quintais ribeirinhos representam uma alternativa sustentável de produção alimentícia e medicinal para as comunidades tradicionais da Amazônia, principalmente no que se refere à diversidade de produtos e à geração de renda. Considerando as funções ecológicas e sociais desses espaços, o objetivo deste trabalho foi analisar a agrobiodiversidade desses ambientes na comunidade Boca do Môa, município de Cruzeiro do Sul, Acre. A coleta dos dados foi realizada através de entrevistas participativas, formais e indutivas seguindo um questionário semiestruturado com questões abertas, além de visitas in loco. Os roçados apresentam diversas espécies na mesma área, sendo a mandioca o principal cultivo. Os quintais possuem arranjos espaciais de espécies alimentícias, destacando-se frutíferas e hortaliças, além de plantas medicinais. Assim, tanto os quintais quanto os roçados auxiliam na subsistência e na renda das famílias ribeirinhas dessa comunidade.

Palavras-chave: Amazônia; Comunidades tradicionais; Recursos vegetais

\section{Abstract}

Agrobiodiversity in riparian backyards and rural cutover lands in the Boca do Moa community Acre, Brazil. The riparian rural cutover lands and backyards represent a sustainable alternative for the production of food and medicinal drugs to the traditional communities from the Amazon, especially with regard to products diversity and income generation. Considering the ecological and social functions of these spaces, this paper aims at analyzing the agrobiodiversity of these environments in the Boca Moa community, in the town of Cruzeiro do Sul, Acre, Brazil. Data collection was carried out through participative, formal, and inductive interviews following a semi-structured questionnaire with open questions, besides in loco visits. The rural cutover lands present many species at the same area, and manioc is the main product cultivated. The backyards have spatial arrangements of food species, with emphasis on fruits and vegetables, besides medicinal plants. Thus, both the backyards and rural cutover lands participate in the subsistence and income of riparian families from this community.

Key words: Amazon; Plant resources; Traditional communities 


\section{Introdução}

Os quintais e roçados da Amazônia apresentam importância para a subsistência dos povos e para o conhecimento sobre os recursos naturais dessas populações. O manejo adaptado a ecossistemas específicos está sendo substituído pela introdução de elementos externos utilizados nos sistemas convencionais de produção vegetal, como produtos sintéticos. Diante da marcha acelerada da urbanização, exploração dos ambientes naturais e das possíveis mudanças culturais, é preciso resgatar o conhecimento que as populações tradicionais detêm sobre a agrobiodiversidade, bem como as formas de cultivo e uso desses recursos naturais (CARNEIRO, 2009).

Segundo a FAO (1999), a agrobiodiversidade consiste na diversificação de plantas, animais e microrganismos utilizados direta ou indiretamente para alimentação e agricultura, incluindo a diversidade dos recursos genéticos e espécies utilizadas para fins medicinais, fibra e combustível. Assim, a agrobiodiversidade não inclui apenas espécies alimentícias, mas também espécies que são utilizadas para fins terapêuticos e matéria-prima. A agrobiodiversidade é gerida por agricultores, sem os quais muitos componentes não sobreviveriam à interferência humana. Nesse contexto, o conhecimento local e a cultura são considerados partes integrantes da gestão da biodiversidade agrícola, pois molda e conserva esta dinâmica relação entre sociedades humanas e as plantas (CONWAY, 1987; FAO, 1999; MARZALL, 2007).

A agrobiodiversidade constitui-se num sistema agrícola, onde se procura associar conservação e manejo com desenvolvimento sustentável (SANTOS, 1996). Essa percepção procura incorporar e adaptar para a Amazônia o conceito e princípios de agroecologia, desenvolvimento local e economia solidária na tentativa de apresentar respostas para comunidades ribeirinhas.

Sabe-se que questões relevantes sobre a biodiversidade são relacionadas com a utilização dos quintais e roçados existentes em zonas rurais, pois estes permitem possibilidades de experimentação, seleção e constituem um rico reservatório de germoplasma, o que contribui para a conservação da diversidade biológica (AMARAL, 2008).
Nesse sentido, o conhecimento local e a cultura podem ser considerados partes integrantes do etnoagrícola e da diversidade, pois é a atividade humana da agricultura que molda e conserva as espécies. Os diversos sistemas agroflorestais como: quintais, roçados e capoeiras enriquecidas são considerados sistemas altamente conservadores da diversidade agrícola e cultural em todas as faixas tropicais do mundo (MACHADO et al., 2008).

O manejo, desenho e função das espécies do local pelos agricultores são os principais aspectos buscados através dos estudos da agrobiodiversidade (DUBOIS et al., 1996). A interação desses recursos com as populações locais está baseada em suas necessidades e, em geral, caracteriza-se por sua heterogeneidade e utilização de tecnologias simples mantendo o equilíbrio ecológico natural.

Desta forma, suas práticas agroecológicas podem identificar um possível diálogo entre o "tradicional" e novas formas de subsistir diante do quadro políticoambiental, utilizando os recursos e os conhecimentos sobre a terra (NODA et al., 2002). A coleta de informações dessas populações é fundamental para se obter e resgatar o conteúdo de aspectos culturais, muitas vezes específicos de cada local e importantes para o uso das plantas (ELISABETSKY; SETZER, 1987).

As ciências que se ocupam de investigar a relação pessoas/plantas estão preocupadas em registrar e conhecer as estratégias e conhecimentos dos povos, procurando também usar essas informações em benefício dessas próprias pessoas (ALBUQUERQUE, 2002). Nesse sentido, o conhecimento da agrobiodiversidade pode revelar o grau de harmonia entre homem-natureza e ainda indicar caminhos para conservação e manejo de espécies (MARTINS, 1994).

Assim, considerando que o agroecossistema é produto da intervenção do agricultor no ambiente, tornase essencial a análise dos aspectos sociais e ecológicos, assim como a percepção dos moradores ribeirinhos ao seu entorno natural. Nesse contexto, o objetivo do presente trabalho foi analisar a agrobiodiversidade através de um levantamento das espécies vegetais (alimentícias e medicinais) encontradas em quintais e 
roçados ribeirinhos na comunidade Boca do Môa, no município de Cruzeiro do Sul, Estado do Acre.

\section{Material e Métodos}

\section{Área de estudo}

A pesquisa foi realizada no município de Cruzeiro do Sul, no Estado do Acre, latitude 07³7'52"S e longitude $72^{\circ} 40^{\prime} 12^{\prime}$ 'W. De acordo com a classificação de Köppen, o clima da região é do tipo equatorial quente e úmido com duas estações bem definidas: uma seca, geralmente de junho a novembro; e outra chuvosa, de dezembro a maio, com índices pluviométricos variando de $1.600 \mathrm{~mm}$ a $2.750 \mathrm{~mm} / \mathrm{ano}$. As temperaturas médias anuais são elevadas, variando entre $24,5^{\circ} \mathrm{C}$ e $32^{\circ} \mathrm{C}$ com $85 \%$ de umidade relativa do ar (ACRE, 2006).

A formação vegetal do município é composta basicamente por floresta tropical aberta (baixos platôs e aluvial) e floresta tropical densa (baixos platôs, superfície dissecada da Serra do Divisor). A região é formada pelas bacias hidrográficas do Juruá e Môa. Apresenta uma grande diversidade de solos, com predominância das classes de Argissolos e Luvissolos (ACRE, 2006).

O município de Cruzeiro do Sul possui uma população de aproximadamente 67.441 habitantes, sendo que $42,5 \%$ vivem na zona rural (IBGE, 2011). Com uma área de $7.781,5 \mathrm{~km}^{2}$, limita-se ao norte com o Estado do Amazonas e ao sul com o Peru. A região é conhecida como "Terra dos Nauas", devido à tribo indígena os Nauas, que habitavam essa região. $\mathrm{Na}$ época das chuvas (novembro a maio) o acesso só é possível por via fluvial ou aérea. A agricultura, pecuária e pesca são as principais atividades econômicas, destacando-se na produção da farinha de mandioca, muito apreciada nos grandes centros urbanos do país e também na criação de gado de corte.

A comunidade Boca do Môa, também conhecida como Seringal Harmonia, é constituída por aproximadamente 90 famílias, que migraram para o local no período do segundo ciclo de exploração da borracha (1942-1945). Historicamente, a região era formada por estradas de seringa, que compreendiam seringais adjacentes ao rio Juruá. O seringal pertencia a Antônio Marques de Menezes, pernambucano excursionista que explorou o rio Môa com o intuito de povoar a região habitada pelos índios Nauas em 1889. A comunidade localiza-se a aproximadamente $3 \mathrm{~km}$ do centro do município (ACRE, 2006).

\section{Coleta dos dados}

Os dados foram coletados no período de agosto de 2010 a junho de 2011 durante cinco visitas na comunidade. Foi realizada a amostragem escolhendo-se como primeiro ponto de entrevista a primeira residência no início da comunidade, sendo que a cada três após o primeiro amostrado iniciava-se uma próxima coleta e assim por diante. As entrevistas foram realizadas in loco nos espaços junto aos moradores, com o objetivo de coletar amostras de plantas e identificar em campo as espécies. Os espaços de produção foram medidos com auxílio de uma trena digital, com o intuito de se obter a área mediana de produção.

As entrevistas foram participativas, formais e indutivas seguindo um questionário semi-estruturado. Questionavam-se os moradores com perguntas abertas sobre o uso dos recursos vegetais, importância dos quintais e roçados, dando liberdade ao informante para responder segundo seus conceitos e palavras próprias. O motivo da conjugação dessas técnicas deveu-se às suas flexibilidades em termos de estrutura, somada à necessidade presencial do pesquisador em fazer alguns esclarecimentos no decorrer da entrevista para a obtenção de aspectos botânicos e ecológicos das plantas utilizadas.

A identificação e a classificação botânica foram realizadas em campo e por comparação de literatura especializada (LORENZI, 2002; LORENZI; MATOS, 2008). Para as espécies não identificadas em campo foram coletadas amostras e identificadas no Herbário da Universidade Federal do Acre - UFAC/Parque Zoobotânico.

\section{Análise dos dados}

Todas as informações coletadas foram transferidas para um banco de dados eletrônico, sendo sistematizadas e processadas. Determinou-se os parâmetros 
fitossociológicos de frequência absoluta $(F A)$ pela expressão $F A=N E / N T$, onde $N E$ significou o número de vezes que uma determinada espécie foi encontrada nos quintais roçados e $N t$ foi o número total de quintais e roçados observados; e a frequência relativa $(F R)$ de cada espécie $F R=F A / N T * 100(\%)$ expressa em porcentagem. Através de cada frequência relativa calculada as espécies foram classificadas em: alta densidade (AD), quando a espécie foi observada em $80 \%$ a $100 \%$ dos quintais e roçados, média densidade (MD), quando a espécie foi observada entre $40 \%$ e $79 \%$; e baixa densidade (BD) quando foi observada abaixo ou igual a $39 \%$ dos quintais e roçados.

Quanto à classificação em categorias de uso foram enquadradas, segundo o pesquisador, nas seguintes: alimentícia, condimentar, medicinal e outros (sombra, madeira). Posteriormente os dados foram transformados em valores percentuais com a intenção de realizar uma análise estatística descritiva das variáveis quantitativas e qualitativas para serem exibidos na forma de gráficos e tabelas.

\section{Resultados e Discussão}

Os ribeirinhos da comunidade Boca do Môa apresentam relações e usos diferentes com os recursos naturais, sendo observado durante as entrevistas uma percepção conservacionista de uso e manutenção das propriedades ambientais. Os quintais e seus roçados em ecossistemas de várzea como para efeito dessa pesquisa, apresentam variedades agrícolas cultivadas em arranjos de sistemas de produção, que são adaptados com o ambiente e o período do ano.

A pesquisa constou de uma intensidade amostral de $25 \%$ sobre o número total de estabelecimentos com espaços de produção na comunidade. Os ribeirinhos entrevistados estão compreendidos numa faixa etária entre 35-82 anos, com idade mediana de 46 anos, sendo 17 homens e 8 mulheres. Estudos realizados no Brasil revelaram que o maior número de trabalhadores da agricultura no meio rural está na faixa de idade entre 40 a 69 anos, e que apenas 2,5\% possuem menos de 30 anos (EMBRAPA, 1998; GALVÃO et al.,1999; QUIRINO et al., 2002). Com relação a suas origens, são todos do
Estado do Acre, nativos dos municípios de Cruzeiro do Sul, Marechal Thaumaturgo, Rodrigues Alves e Porto Walter, sendo descendentes de indígenas ou seringueiros.

A ocupação do seringal Boca do Môa tem sua origem com a vinda de muitos extrativistas, agricultores e pescadores que residiam em outras localidades e migraram para a região em busca de melhores oportunidades. A localização próxima ao porto fluvial do município de Cruzeiro do Sul facilitaria a exportação da borracha, além do acesso ao serviço de saúde pública, principalmente no tratamento de doenças tropicais como a malária. Dessa forma, após o declínio definitivo do ciclo da borracha e a adesão de toda a área à União, muitos ex-seringueiros permaneceram na ocupação e construíram suas moradias, tendo acesso pela primeira vez à propriedade privada, iniciando a atividade agrícola de subsistência nos seus roçados e quintais.

Quanto ao nível de escolaridade 55\% possuem o Ensino Fundamental Incompleto da $1^{\mathrm{a}}$ à $8^{\mathrm{a}}$ série, Ensino Médio (15\%), analfabetos (20\%) e 10\% com Ensino Superior. A taxa de analfabetismo e o baixo nível de escolaridade refletem o histórico de décadas passadas onde o acesso à educação era restrito, principalmente do interior do Acre. Além disso, muitos jovens priorizam as tarefas domésticas, o trabalho na roça e nas cidades para complementar a renda familiar. Muitos jovens possuem condições de estudar, mas o problema passa a ser o acesso à sala de aula, principalmente pela distância do local, ainda um problema na Amazônia.

No que se refere à religiosidade, os informantes são católicos $(85 \%)$ e protestantes $(15 \%)$. Alguns católicos também participam de manifestações religiosas como o Santo-Daime. Contudo, não foi observada nos quintais e roçados a espécie Banisteria caapi Spruce ex Griseb., utilizada nos rituais, pois segundo os moradores a mesma encontra-se no interior da floresta.

O tempo médio de moradia dos entrevistados na comunidade foi de 18 anos, sendo o mínimo de seis e o máximo de 62 anos, o morador mais antigo. O tempo de permanência no local pode apresentar relação diretamente proporcional à diversidade florística de espécies encontradas nos quintais, principalmente em relação às plantas medicinais. 
Os ribeirinhos dessa comunidade vivem principalmente dos roçados de mandioca, do extrativismo vegetal, pesca para autoconsumo e comercialização em pequena escala, quintais com frutas e hortaliças e da criação de pequenos animais domésticos. Os principais produtos agrícolas por ordem de importância alimentar na comunidade são: mandioca, milho, feijão e arroz. Essas culturas fazem parte da alimentação diária desses ribeirinhos. Nesse sistema agroambiental predomina a cultura da mandioca, sendo cultivada em $90 \%$ dos espaços visitados. É utilizada na alimentação e fabricação de subprodutos como a farinha de mandioca, farinha de tapioca, goma e beiju. Observação diferente foi encontrada por Pasa e Avila (2010) na parte alta da bacia do rio Arareau em Mato Grosso, onde poucas pessoas possuíam plantações de mandioca para a produção de farinha e derivados.

A produção agrícola é para o consumo da casa e o excedente é transportado através de barcos para comercialização na feira às margens do rio Juruá a 3 $\mathrm{km}$ da comunidade. Foi observado na área pesquisada, que os roçados de mandioca têm papel importante para os ribeirinhos da comunidade Boca do Môa, pois são os principais produtos alimentares tanto para a subsistência quanto para a comercialização na forma de farinha de mandioca.

Os roçados na região são conhecidos como áreas para cultivo de mandioca, milho e feijão. Apresentam uma área mediana de $13.500 \mathrm{~m}^{2}$ e são feitos a partir do corte e queima da vegetação, sendo utilizada a enxada no preparo do solo. O preparo da área ocorre entre os meses de março a abril, sendo que $35 \%$ dos entrevistados contratam mão de obra externa para auxiliar na atividade. A semeadura inicia-se no mês de maio e a colheita nos meses de outubro a fevereiro.

Para o plantio de mandioca as manivas são oriundas do próprio local ou trazidas de outros municípios e plantadas após a cheia das águas. Além da mandioca, observou-se a introdução de culturas como o milho e o feijão na mesma área, além de algumas frutíferas como o mamão e o abacaxi. Para Machado et al. (2008) os principais aspectos da agrobiodiversidade estão relacionados com: segurança alimentar, composição da renda, conservação de recursos genéticos e da diversidade cultural associada às populações locais e povos indígenas. Assim, a produção destina-se na sua maioria para a subsistência e o excedente para a venda, auxiliando na renda da família.

Segundo os entrevistados a escolha da área para implantação dos roçados se dá nas proximidades da floresta e dos rios (Figura 1), pois o índice de pragas e doenças é menor, além de solos mais férteis. Quando questionados sobre derrubadas e queimadas para plantio diziam que se referia à área de preservação permanente e que as terras já antropizadas são suficientes para a produção de alimentos para a família e os animais.

$\mathrm{O}$ cultivo em praias, que se formam às margens dos rios Juruá e Môa é prática anual da comunidade. Essas áreas são enriquecidas com nutrientes quando

FIGURA 1: Plantio de milho (esquerda) e roçados às margens do rio Môa (direita), comunidade Boca do Môa, Cruzeiro do Sul, Acre.

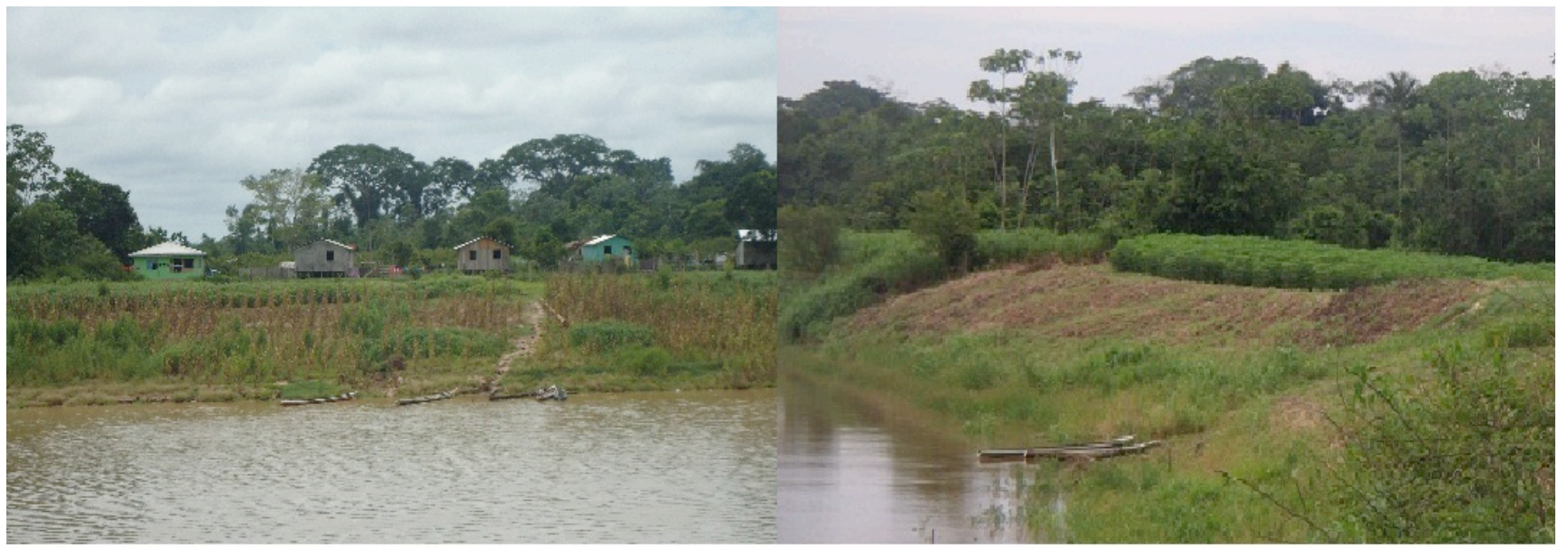


as águas sobem com a chuva do inverno (outubro a março), cultivando-se milho, feijão, arroz e melancia. Têm sua importância na alimentação da família, além de uma fonte de renda alternativa. As praias escolhidas são as próximas à residência, sendo que em $15 \%$ dos espaços observados, as mulheres são responsáveis pela implantação e manejo do cultivo. Das culturas identificadas nas margens dos rios, a melancia apresentou maior expressão (80\%), seguida do feijão (75\%), arroz $(60 \%)$ e milho $(40 \%)$.

Quanto aos quintais visitados, apresentaram uma área mediana de $1400 \mathrm{~m}^{2}$ e todos se localizavam na parte de trás das residências. $\mathrm{O}$ mesmo foi verificado por Pasa e Avila (2010) e por Pasa et al. (2005). As hortas utilizadas para cultivo de hortaliças são pequenas, suspensas com madeira e construídas a partir de resíduos florestais, manejando-se de forma orgânica sem o uso de agrotóxicos e adubos químicos. A produção é destinada somente para a subsistência da família, e a mão de obra é familiar, principalmente de mulheres.

Durante a pesquisa, verificou-se nos quintais que os entrevistados têm o costume de cultivar hortaliças em canteiros suspensos e separados (Figura 2), uma vez que essas plantas exigem maiores cuidados quanto ao solo, geralmente mal drenado, luminosidade e intensidade das chuvas invernais. Além disso, com a enchente dos rios nos meses de janeiro a abril não ocorre perda desses materiais por inundação. Algumas vezes, essas plantas também são cultivadas em conjunto com plantas medicinais, assim ao utilizarem tais técnicas, os moradores podem facilitar o manejo e o controle de pragas e doenças que podem ocasionar danos às plantas.

Nos fundos dos quintais predomina o cultivo de um pequeno pomar que atende à demanda familiar. A maioria das frutíferas encontradas é nativa da região e foram preservadas servindo de complemento para a alimentação. Os quintais na comunidade Boca do Môa apresentam características semelhantes às descritas por Noda et al. (2002) por apresentarem uma área relativamente grande, seus espaços são destinados ao manejo de árvores, arbustos e ervas, para diferentes finalidades, com cultivos perenes e anuais, cultivados em consórcio com pequenos animais.

Identificou-se um total de 54 espécies vegetais (Tabela 1), sendo distribuídas em 27 famílias botânicas, destacando-se: Solonaceae, Lamiaceae, Arecaceae, Asteraceae, Cucurbitaceae e Fabaceae. Das 54 espécies 35 são utilizadas como alimentícias, principalmente as famílias: Alliaceae, Brassicaceae, Fabaceae e Solanaceae. Lourenço et al. (2009), pesquisando quintais em assentamentos na Amazônia Central, encontrou que $73 \%$ são espécies para uso alimentar, 16\% são usadas como condimentos e típicas de hortas caseiras.

FIGURA 2: Canteiros de hortaliças suspensos na comunidade Boca do Môa, Cruzeiro do Sul, Acre.

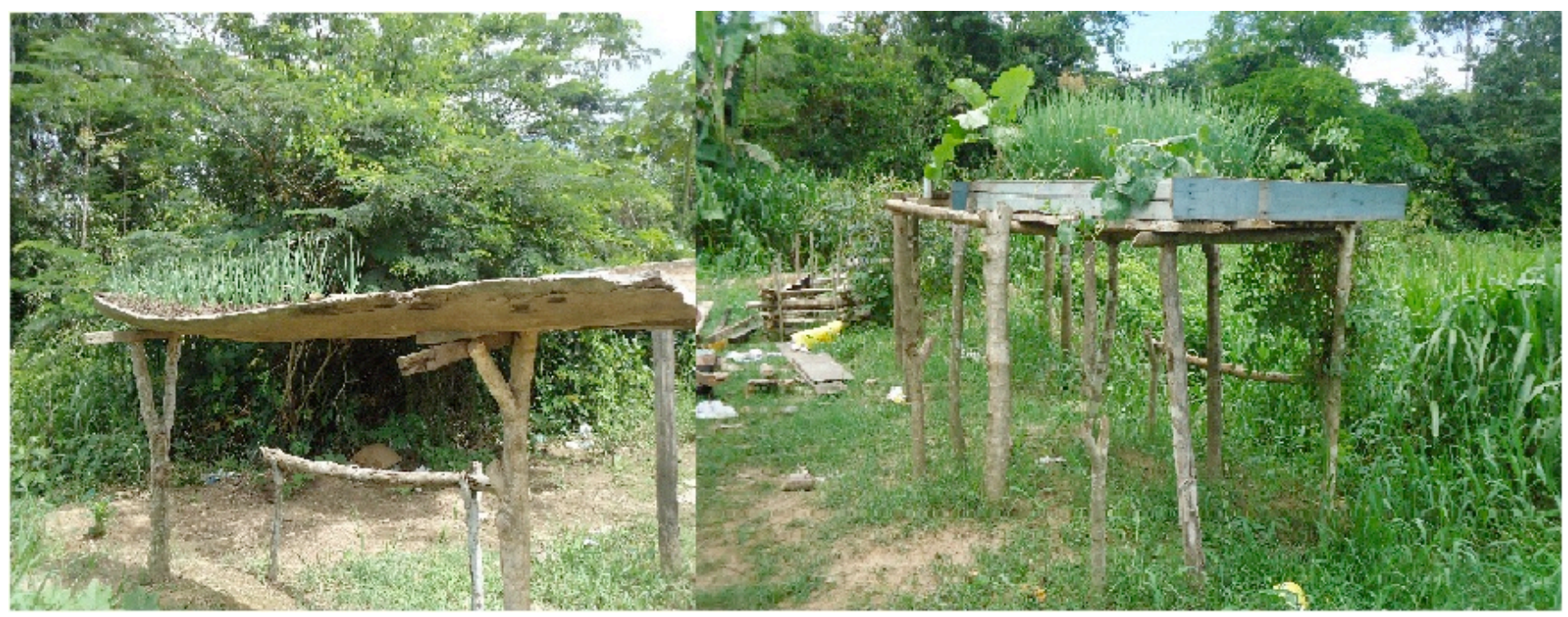


TABELA 1: Famílias e espécies encontradas nos roçados e quintais da comunidade Boca do Môa, Cruzeiro do Sul - Acre.

\begin{tabular}{|c|c|c|c|c|c|}
\hline Família botânica/Espécie & Nome popular & FA & FR & Densidade & Categoria \\
\hline \multicolumn{6}{|l|}{ Alliaceae } \\
\hline Allium schoenoprosum L. & Cebolinha & 15 & 75 & MD & $\mathrm{A} / \mathrm{C}$ \\
\hline Allium сера $\mathrm{L}$. & Cebola & 1 & 5 & $\mathrm{BD}$ & $\mathrm{A} / \mathrm{C}$ \\
\hline \multicolumn{6}{|l|}{ Amaranthaceae } \\
\hline Alternanthera brassiliana Kuntze. & Terramicina & 1 & 5 & $\mathrm{BD}$ & $\mathrm{M}$ \\
\hline Chenopodium ambrosioides L. & Mastruz & 12 & 60 & MD & $\mathrm{M}$ \\
\hline \multicolumn{6}{|l|}{ Anacardiaceae } \\
\hline Anacardium occidentale L. & Cajú & 2 & 10 & $\mathrm{BD}$ & $\mathrm{A} / \mathrm{M}$ \\
\hline Spodias mombin L. & Cajá & 6 & 30 & $\mathrm{BD}$ & $\mathrm{A}$ \\
\hline Mangifera indica $\mathrm{L}$. & Manga & 7 & 35 & $\mathrm{BD}$ & $\mathrm{A}$ \\
\hline \multicolumn{6}{|l|}{ Annonaceae } \\
\hline Annona muricata L. & Graviola & 5 & 25 & $\mathrm{BD}$ & $\mathrm{A} / \mathrm{M}$ \\
\hline \multicolumn{6}{|l|}{ Apiaceae } \\
\hline Coriandrum sativum L. & Coentro & 15 & 75 & MD & $\mathrm{A} / \mathrm{C}$ \\
\hline \multicolumn{6}{|l|}{ Apocynaceae } \\
\hline Aspidosperma nitidum Benth. Ex Mull & Carapanaúba & 2 & 10 & $\mathrm{BD}$ & $\mathrm{M}$ \\
\hline \multicolumn{6}{|l|}{ Arecaceae } \\
\hline Euterpe precatoria Martius & Açaí & 6 & 30 & $\mathrm{BD}$ & $\mathrm{A} / \mathrm{M}$ \\
\hline Oenocarpus bacaba Mart. & Bacaba & 4 & 20 & $\mathrm{BD}$ & $\mathrm{A}$ \\
\hline Mauritia flexuosa $\mathrm{L}$. & Buriti & 12 & 60 & MD & $\mathrm{A}$ \\
\hline \multicolumn{6}{|l|}{ Asteraceae } \\
\hline Lactuca sativa $\mathrm{L}$. & Alface & 4 & 20 & $\mathrm{BD}$ & $\mathrm{A}$ \\
\hline Vernonia polyanthes Less. & Assa-peixe & 3 & 15 & $\mathrm{BD}$ & $\mathrm{M}$ \\
\hline Baccharis trimera ( Less) DC. & Carqueja & 9 & 45 & MD & $\mathrm{M}$ \\
\hline \multicolumn{6}{|l|}{ Asphodelaceae } \\
\hline Aloe vera (L.) Burm. F. & Babosa & 7 & 35 & $\mathrm{BD}$ & $\mathrm{M}$ \\
\hline \multicolumn{6}{|l|}{ Bignoniaceae } \\
\hline Fridericia chica L. G. Lohamann & Crajirú & 13 & 65 & MD & $\mathrm{M}$ \\
\hline \multicolumn{6}{|l|}{ Brassicaceae } \\
\hline Nasturtium officinale R. Br. & Agrião & 4 & 20 & $\mathrm{BD}$ & $\mathrm{M}$ \\
\hline Brassica oleracea L. & Couve & 15 & 75 & MD & $\mathrm{A}$ \\
\hline \multicolumn{6}{|l|}{ Bromeliaceae } \\
\hline Ananas comosus (L.) Merr. & Abacaxi & 6 & 30 & $\mathrm{BD}$ & $\mathrm{A}$ \\
\hline \multicolumn{6}{|l|}{ Caricaceae } \\
\hline Carica papaya $\mathrm{L}$. & Mamão & 5 & 25 & $\mathrm{BD}$ & $\mathrm{A}$ \\
\hline \multicolumn{6}{|l|}{ Cucurbitaceae } \\
\hline Cucurbita pepo L. & Abóbora & 13 & 65 & MD & $\mathrm{A}$ \\
\hline Cucumis sativus L. & Pepino & 7 & 35 & $\mathrm{BD}$ & $\mathrm{A}$ \\
\hline Citrullus vulgaris Schrad & Melancia & 16 & 80 & $\mathrm{AD}$ & $\mathrm{A}$ \\
\hline \multicolumn{6}{|l|}{ Dioscoreaceae } \\
\hline Dioscorea sp. & Cará & 2 & 10 & $\mathrm{BD}$ & A \\
\hline \multicolumn{6}{|l|}{ Euphorbiaceae } \\
\hline Manihot esculenta Crantz. & Mandioca & 18 & 90 & $\mathrm{AD}$ & $\mathrm{A}$ \\
\hline Hevea brasiliensis Müll & Seringueira & 8 & 40 & MD & $\mathrm{O}$ \\
\hline \multicolumn{6}{|l|}{ Fabaceae } \\
\hline Vataireopsis araroba (Aguiar) Ducke & Amargoso & 3 & 15 & $\mathrm{BD}$ & $\mathrm{M}$ \\
\hline Phaseolus vulgaris $\mathrm{L}$. & Feijão & 15 & 75 & MD & $\mathrm{A}$ \\
\hline Hymenaea courbaril L. & Jatobá & 3 & 15 & $\mathrm{BD}$ & $\mathrm{M}$ \\
\hline
\end{tabular}




\begin{tabular}{|c|c|c|c|c|c|}
\hline \multicolumn{6}{|l|}{ Lamiaceae } \\
\hline Hyptis sp. & Hortelã & 3 & 15 & $\mathrm{BD}$ & $\mathrm{M}$ \\
\hline Lippia Alba (Mill) N. E. Br & Erva-cidreira & 3 & 15 & $\mathrm{BD}$ & $\mathrm{M}$ \\
\hline Ocimum basilicum $\mathrm{L}$. & Alfavaca & 8 & 40 & MD & $\mathrm{M}$ \\
\hline Venonia condensata Baker & Boldo & 14 & 70 & MD & $\mathrm{M}$ \\
\hline \multicolumn{6}{|l|}{ Moraceae } \\
\hline Arthocarpus integrifolia $\mathrm{L}$. & Jaca & 2 & 10 & $\mathrm{BD}$ & A \\
\hline \multicolumn{6}{|l|}{ Musaceae } \\
\hline Musa paradisiaca L. & Banana & 16 & 80 & $\mathrm{AD}$ & A \\
\hline \multicolumn{6}{|l|}{ Myrtaceae } \\
\hline Eugenia stipitata Mc Vaugh & Araçá-boi & 3 & 15 & $\mathrm{BD}$ & A \\
\hline Psidium guajava $\mathrm{L}$. & Goiaba & 9 & 45 & $\mathrm{BD}$ & $\mathrm{A}$ \\
\hline \multicolumn{6}{|l|}{ Oxalidaceae } \\
\hline Averrhoa carambola $\mathrm{L}$. & Carambola & 5 & 25 & $\mathrm{BD}$ & A \\
\hline \multicolumn{6}{|l|}{ Pedaliaceae } \\
\hline Sesamum orientale L. & Gergelim & 2 & 10 & $\mathrm{BD}$ & $\mathrm{M} / \mathrm{C}$ \\
\hline \multicolumn{6}{|l|}{ Poaceae } \\
\hline Oryza sativa $L$ & Arroz & 12 & 60 & MD & A \\
\hline Cymbopogon citratus (DC.) Stapf & Capim-cidreira & 1 & 5 & $\mathrm{BD}$ & $\mathrm{M}$ \\
\hline Saccharum officinarum L. & Cana de açúcar & 6 & 30 & $\mathrm{BD}$ & $\mathrm{A}$ \\
\hline Zea mays L. & Milho & 8 & 40 & MD & $\mathrm{A}$ \\
\hline \multicolumn{6}{|l|}{ Rutaceae } \\
\hline Citrus aurantium L. & Laranja & 3 & 15 & $\mathrm{BD}$ & $\mathrm{A}$ \\
\hline Citrus limonum L. & Limão & 2 & 10 & $\mathrm{BD}$ & $\mathrm{A} / \mathrm{M}$ \\
\hline Ruta graveolens L. & Arruda & 1 & 5 & $\mathrm{BD}$ & $\mathrm{M}$ \\
\hline \multicolumn{6}{|l|}{ Solanaceae } \\
\hline Capsicum annuиm L. & Pimentão & 5 & 25 & $\mathrm{BD}$ & A \\
\hline Capsicum chinense Jacq. & $\begin{array}{l}\text { Pimenta } \\
\text { de cheiro }\end{array}$ & 17 & 85 & $\mathrm{AD}$ & $\mathrm{A} / \mathrm{C}$ \\
\hline Capsicum frutescens L. & Pimenta malagueta & 13 & 65 & MD & $\mathrm{C}$ \\
\hline Capsicum sp. & Pimenta doce & 18 & 90 & $\mathrm{AD}$ & $\mathrm{A} / \mathrm{C}$ \\
\hline Lycopersicum esculentum L. & Tomate & 4 & 20 & $\mathrm{BD}$ & $\mathrm{A}$ \\
\hline \multicolumn{6}{|l|}{ Zinziberaceae } \\
\hline Zingiber officinale Roscoe & Gengibre & 8 & 40 & MD & $\mathrm{A} / \mathrm{M}$ \\
\hline
\end{tabular}

Legenda: $\mathrm{A}=\mathrm{Alimentar} ; \mathrm{M}=$ Medicinal; $\mathrm{C}=$ Condimentar; $\mathrm{O}=$ Outros; $\mathrm{AD}=$ Alta densidade; $\mathrm{MD}=$ Média densidade; $\mathrm{BD}=\mathrm{Baixa}$ densidade.

Quanto às medicinais, 21 espécies são utilizadas no tratamento de doenças, sobressaindo as famílias Lamiaceae e Asteraceae. Estas famílias frequentemente são encontradas como as mais representativas em outros estudos etnobotânicos no país, como em Pasa (2004), Santos (2004), Amaral (2008) e Delunardo (2010). De acordo com Souza Brito (1993), essas duas famílias apresentaram espécies medicinais de ampla ocorrência no Brasil, correspondendo a aproximadamente $20 \%$ do total de ervas pesquisadas.

Quanto à categoria condimentar foram identificadas sete espécies que são utilizadas no preparo de alimentos, distribuindo-se nas famílias Alliaceae, Apiaceae, Solanaceae e Pedaliaceae. As espécies mais frequentes (Tabela 1) nos quintais na categoria alimentar foram banana $(\mathrm{FR}=80 \%)$, melancia $(80 \%)$, buriti $(60 \%)$ e goiaba (45\%).

Dentre as hortaliças (Figura 3), destacam-se a pimenta-doce, pimenta-de-cheiro, cebolinha, couve, coentro, abóbora e pimenta-malagueta. A pimenta-doce apresentou (Tabela 1) frequência relativa de $90 \%$ e a pimenta-de-cheiro de $85 \%$, ambas com alta densidade. Verificou-se uma preferência dos ribeirinhos pelo plantio de pimentas, sendo encontrada em todos os quintais, 
além disso, observaram-se diversas espécies do gênero Capsicum na comunidade.

Pasa (2004) salienta que a categoria de uso de uma espécie vegetal pode ser cumulativa, uma espécie pode ser utilizada para a alimentação e ser medicinal. Neste estudo, a multiplicidade de usos está representada por 11 espécies, como por exemplo, cebolinha, cebola, cajú, graviola, açaí e gengibre.

Na categoria medicinal (Figura 4), destacaram-se o boldo (70\%), o crajiru (65\%), o mastruz (60\%), seguidos da carqueja (45\%), da alfavaca $(40 \%)$, do gengibre $(40 \%)$ e da babosa (35\%).

FIGURA 3: Principais plantas hortícolas encontradas nos quintais ribeirinhos na comunidade Boca do Môa, Cruzeiro do Sul, Acre.

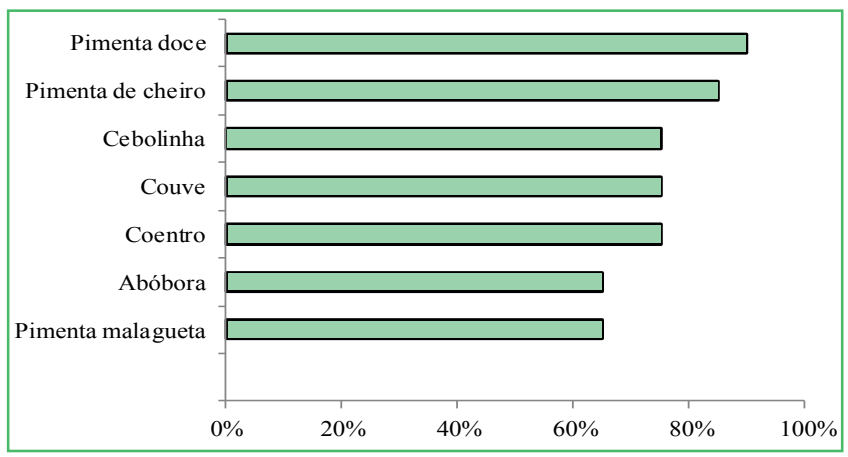

FIGURA 4: Principais plantas medicinais encontradas nos quintais ribeirinhos na comunidade Boca do Môa, Cruzeiro do Sul, Acre.

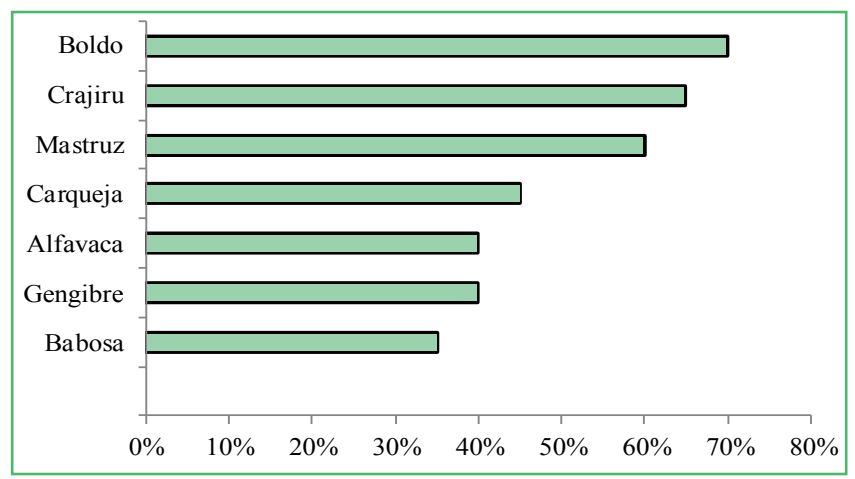

Os ribeirinhos da comunidade Boca do Môa utilizam sistemas de produção com características semelhantes às das demais localidades de várzea na Amazônia, com influência das enchentes e da proximidade do centro urbano. Seus roçados e quintais apresentam uma diversidade de espécies vegetais úteis, as quais são utilizadas e manejadas pelos agricultores para garantir a oferta de produtos alimentícios e medicinais para subsistência e geração de renda. Esses espaços demonstram a importância da conservação e preservação da biodiversidade em locais de várzea e manutenção das tradições culturais de plantio em quintais e roçados na Amazônia.

\section{Agradecimentos}

Os autores agradecem a todos os moradores da comunidade Boca do Môa pela recepção nos trabalhos a campo e ao CNPq pela concessão da bolsa de estudos.

\section{Referências}

ACRE - Governo do Estado do Acre. Programa Estadual de Zoneamento Ecológico Econômico. Zoneamento EcológicoEconômico do Acre, Fase II, Documento síntese - escala 1:250.000. Rio Branco: SEMA, 2006. 355 p.

AMARAL, C. N. Recursos vegetais dos tradicionais quintais de Rosário Oeste - Mato Grosso. 2008. 80 f. Dissertação (Mestrado em Ecologia e Conservação da Biodiversidade) - Universidade Federal de Mato Grosso, Cuiabá. 2008.

ALBUQUERQUE, U. P. Introdução a etnobotânica. Recife: Bagaço, 2002. 87 p.

CONWAY, G. The properties of agroecosystems. Agricultural Systems, Essex, v. 24, n. 2, p. 95-117, 1987.

CARNEIRO, M. R. B. A flora medicinal no centro oeste do Brasil: um estudo de caso com abordagem etnobotânica em Campo Limpo de Goiás. 2009. 243 f. Dissertação (Mestrado em Sociedade, Tecnologia e Meio Ambiente) - Universidade Evangélica de Goiás, Anápolis. 2009.

DELUNARDO, T., A. A agrobiodiversidade em quintais urbanos de Rio Branco, Acre. 2010. 112 f. Dissertação (Mestrado em Agronomia) - Universidade Federal do Acre, Rio Branco. 2010. DUBOIS, J.; VIANA, V. M.; ANDERSON, A. B. Manual agroflorestal para a Amazônia. v.1. Rio de Janeiro: REBRAF, 1996. $28 \mathrm{p}$.

ELISABETSKY, E.; SETZER, R. Caboclo concepts of disease, diagnosis, and therapy: implications for ethnofarmacology and health systems in Amazonia. In: PARKER, E. P. (Ed.). The amazon caboclo: historical and contemporany perspectives. Williamsburg: Studies on Third World Societies, 1987. p. 243-278.

EMBRAPA. Centro Nacional de Pesquisa de Florestas (Colombo, $P R)$. Caracterização de sistemas de uso da terra e planejamento de ações para melhoria do sistema agroflorestal da erva-mate (Ilex paraguariensis St. Hil.) no município de Matão Leitão, RS. Colombo: EMBRAPACNPF, Documento, 30, 1998. 31 p.

FAO. Sustaining agricultural diversity in agro-ecosystems functions. Roma: Italy, 1999. $43 \mathrm{p}$.

GALVÃO, E. U. P.; CONTO, A. J.; HOMMA, A. K. O.; OLIVEIRA, R. F.; CARVALHO, R. A.; FERREIRA, C. A. P.; MENEZES, 
A. J. E. A. Introdução de mudanças tecnológicas em sistemas de produção familiares: o caso da associação dos pequenos e microprodutores rurais do Panela - Irituia - Pará. Belém: Embrapa Amazônia Oriental, Documento 17, 1999. 64 p.

IBGE - Instituto Brasileiro de Geografia de Estatística. Informações Estatísticas. 2011. Disponível em $<$ http://www.ibge. gov.br/cidadesat/default.php>.

LORENZI, H. Árvores brasileiras: manual de identificação e cultivo de plantas arbóreas do Brasil. v. 2. Nova Odessa: Instituto Plantarum, 2002. 384 p.

LORENZI, H.; MATOS, F. J. A. Plantas medicinais do Brasil: nativas e exóticas cultivadas. Nova Odessa: Instituto Plantarum, 2008. 244 p.

LOURENÇO, J. N. P.; SOUSA, S. G. A.; WANDELLI, E. V.; LOURENÇO, F. S.; GUIMARÃES, R. R.; CAMPOS, L. S.; SILVA, R. L.; MARTINS, V. F. C. Agrobiodiversidade nos quintais agroflorestais em três assentamentos na Amazônia central. Revista Brasileira de Agroecologia, Porto Alegre, v. 4, n. 2, p. 965-969, 2009

MACHADO, A. T.; SANTILli, J.; MAGALHÃES, R. A. Agrobiodiversidade com enfoque agroecológico: implicações conceituais e jurídicas. Brasília: Embrapa Informação Tecnológica; Embrapa- Secretaria de Gestão e Estratégia, 2008. 98 p.

MARTINS, P. S. Biodiversity and agriculture: patterns of domestication of Brazilian native plant species. Academia Brasileira de Ciências, Rio de Janeiro, v. 66, n. 1, p. 219-224, 1994.

MARZALL, K. Fatores geradores da agrobiodiversidade Influências socioculturais. Revista Brasileira de Agroecologia, Porto Alegre, v. 2, n. 1, p. 237-240, 2007.
NODA, S.; NODA, H.; MARTINS, A. L. U. Papel do processo produtivo tradicional na conservação dos recursos genéticos vegetais. In: RIVAS A.; FREITAS, C. E. C. (Org.). Amazônia uma perspectiva interdisciplinar. Manaus: Editora da Universidade do Amazonas, 2002. p. 155-178.

PASA, M. C. Etnobiologia de uma comunidade ribeirinha no alto da bacia do rio Aricá-Açú, Cuiabá, Mato Grosso, Brasil. 2004. 174 f. Tese (Doutorado em Ciências) - Universidade Federal de São Carlos, São Carlos. 2004.

PASA, M. C.; SOARES, J. J.; GUARIM-NETO, G. Estudo etnobotânico na comunidade de Conceição-Açu (alto da bacia do rio Aricá-Açu, MT, Brasil). Acta Botânica Brasílica, São Paulo, v. 19, n. 2, p. 195-207, 2005.

PASA, M. C.; AVILA, G. Ribeirinhos e recursos vegetais: a etnobotânica em Rondonópolis, Mato Grosso, Brasil. Interações, Campo Grande, v. 11, n. 2, p. 195-204, 2010.

QUIRINO, T. R.; GARAGORRY, F. L.; SOUSA, C. P. Diagnóstico sociotécnico da agropecuária brasileira: I Produtores. Brasília: Embrapa Informação Tecnológica, 2002. 59 p.

SANTOS, J. C. Análise da rentabilidade, sob condições de risco, de um sistema agroflorestal adotado por pequenos produtores de cacau na região da Transamazônica, Pará. 1996. 128 f. Dissertação (Mestrado em Economia Rural) - Universidade Federal do Ceará, Fortaleza. 1996.

SANTOS, S. Um estudo etnoecológico dos quintais de Alta Floresta-MT. 2004. 98 f. Dissertação (Mestrado em Ecologia e Conservação da Biodiversidade) - Universidade Federal de Mato Grosso, Cuiabá. 2004.

SOUZA BRITO, A. R. M.; SOUZA BRITO, A. A. Forty years of Brazilian medicinal plant research. Journal of Ethnopharmacology, Shannon, v. 39, p. 53-67, 1993. 